\title{
Analysis, Synthesis, and Classification of Nonlinear Systems Using Synchronized Swept-Sine Method for Audio Effects
}

\author{
Antonin Novak, Laurent Simon, and Pierrick Lotton \\ Laboratoire d'Acoustique, Université du Maine, UMR CNRS 6613, 72000 Le Mans, France \\ Correspondence should be addressed to Antonin Novak, ant.novak@gmail.com \\ Received 1 March 2010; Revised 16 June 2010; Accepted 5 July 2010 \\ Academic Editor: Mark Sandler
}

Copyright (C) 2010 Antonin Novak et al. This is an open access article distributed under the Creative Commons Attribution License, which permits unrestricted use, distribution, and reproduction in any medium, provided the original work is properly cited.

\begin{abstract}
A new method of identification, based on an input synchronized exponential swept-sine signal, is used to analyze and synthesize nonlinear audio systems like overdrive pedals for guitar. Two different pedals are studied; the first one exhibiting a strong influence of the input signal level on its input/output law and the second one exhibiting a weak influence of this input signal level. The Synchronized Swept Sine method leads to a Generalized Polynomial Hammerstein model equivalent to the pedals under test. The behaviors of both pedals are illustrated through model-based resynthesized signals. Moreover, it is also shown that this method leads to a criterion allowing the classification of the nonlinear systems under test, according to the influence of the input signal levels on their input/output law.
\end{abstract}

\section{Introduction}

Various classical analog audio effects fall into the category of nonlinear effects such as compression, harmonic excitation, overdrive, or distortion for guitars. Digital emulations of nonlinear audio effects can be obtained when using a suitable nonlinear model. Such nonlinear models are available in the literature, for example, Volterra model [1], neural network model [2], MISO model [3], NARMAX model [4], hybrid genetic algorithm [5], extended Kalman filtering [6], or particle filtering [7].

A new method for the identification of nonlinear systems, based on an input exponential swept-sine signal has been proposed by Farina et al. [8,9]. This method has been recently modified for the purpose of nonlinear model estimation [10] and allows a robust and fast onepath analysis and identification of the unknown nonlinear system under test. The method is called Synchronized Swept Sine method as it uses a synchronized swept sine signal for identification.

A nonlinear effect can be modeled either by a simple static nonlinear input/output law, where each input amplitude is directly mapped to an output amplitude (nonlinear system without memory), or on a more complex way by nonlinear laws which take memory into account, meaning that the memoryless nonlinearities and the linear filtering are mixed. Moreover, several nonlinear audio effects include amplifiers, the gain of which is automatically controlled by the level of the input signal [11]. In other words, the performance of nonlinear systems with memory may also depend on parameters of the input signal, such as its level or its past extrema, as for the hysteretic systems [12].

This classification of nonlinear systems according to the influence of the input signal parameters on the input/output law leads to a similar classification of the identification methods. The methods for identification of static nonlinearities indeed do not require the same level of model complexity as methods used for nonlinear systems with memory or with gain control.

In this paper, it is shown that the Synchronized Swept Sine method is suited to analyze, classify, and synthesize the nonlinear systems under test. In the frame of this work, two different overdrive pedals have been tested; the first one exhibiting a strong influence of the input signal level on its input/output law and the second one exhibiting a weak influence of this input signal level. 
In Section 2, Synchronized Swept Sine method is shortly presented. This method leads to a nonlinear model (Section 3), made up of several branches, each branch consisting of a nonlinear function and a linear filter. The nonlinear functions are chosen as a power series that makes the model equivalent to a Generalized Polynomial Hammerstein (GPH) model. Next, the measurements on overdrive pedals are presented in Section 4. The behaviors of both systems are illustrated through model-based resynthesized signals. Finally, in Section 5, we propose a criterion based on the GPH model to classify the nonlinear systems according to the importance of the influence of the input signal parameters on the input/output law of the system under test.

\section{Analysis of Nonlinear Systems}

The nonlinear system identification method used in this paper is based on an excitation by a swept-sine signal (also called chirp) exhibiting an exponential instantaneous frequency $f_{i}(t)$. This so-called Synchronized Swept-Sine method allows the identification of a system in terms of harmonic distortion at several orders. This identification is conducted in several steps.

First, an exponential swept-sine signal $x_{s}(t)$ is generated and used as the input signal of the nonlinear system under test. The excitation swept-sine signal $x_{s}(t)$ is defined as

$$
x_{s}(t)=A_{s} \sin \left\{2 \pi L\left[\exp \left(\frac{f_{1} t}{L}\right)-1\right]\right\} \text {, }
$$

where

$$
L=\operatorname{Round}\left(\frac{\widehat{T} f_{1}}{\ln \left(f_{2} / f_{1}\right)}\right)
$$

$f_{1}$ and $f_{2}$ being start and stop frequencies, and $\hat{T}$ being the time length of the swept-sine signal. The rounding operator is necessary to synchronize the swept-sine signal for higher-order contributions with linear component as depicted in Figure 1. This condition is necessary for the model identification and for a proper reconstruction of the output signal.

Then, the distorted output signal $y_{s}(t)$ of the nonlinear system is recorded for use in the so-called nonlinear convolution [8]. Next, the signal denoted $\tilde{x}_{s}(t)$ is derived from the input signal $x_{s}(t)$ as its time-reversed replica with amplitude modulation in such a way that the convolution between $x_{s}(t)$ and $\tilde{x}_{s}(t)$ gives a Dirac delta function $\delta(t)$. The signal $\tilde{x}_{s}(t)$ is called the inverse filter [8].

Finally, the convolution between the output signal $y_{s}(t)$ and the inverse filter $\tilde{x}_{s}(t)$ is performed, leading to

$$
y_{s}(t) * \tilde{x}_{s}(t)=\sum_{i=1}^{\infty} h_{i}\left(t+\Delta t_{i}\right)
$$

where $h_{i}(t)$ are the higher-order impulse responses and $\Delta t_{i}$ are the time lags between the first and the $i$ th impulse response. Since the result of convolution $y_{s}(t) * \tilde{x}_{s}(t)$ consists of a set of higher-order impulse responses that are time

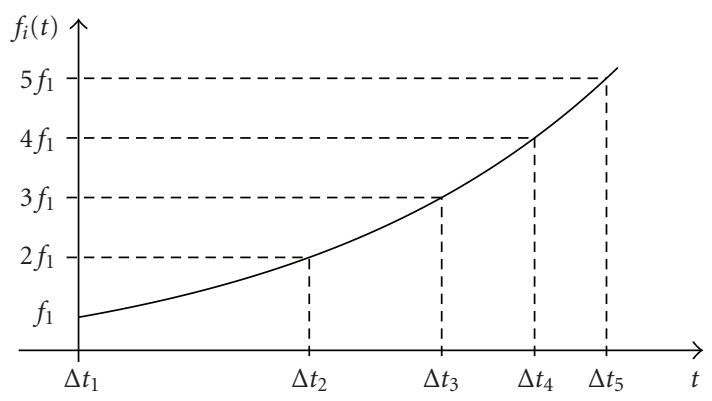

(a)

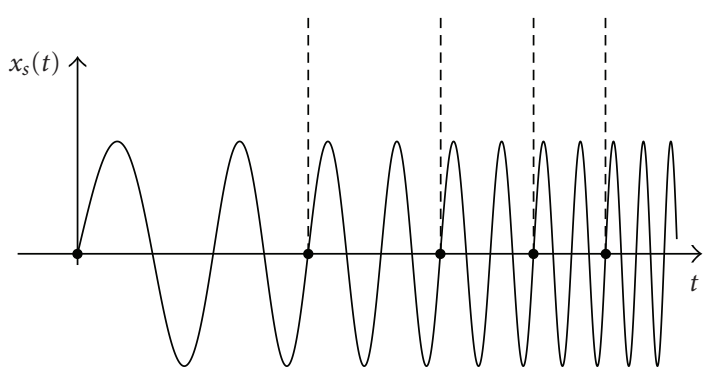

(b)

FIGURE 1: Swept-sine signal $x_{s}(t)$ in the time domain (b), with the time length chosen according to the instantaneous frequency $f_{i}(t)$ (a).

shifted, each partial impulse response can be separated from each other.

The set of higher-order nonlinear impulse responses $h_{i}(t)$ can also be expressed in the frequency domain. The frequency response functions of the higher-order nonlinear impulse responses $h_{i}(t)$ are then defined as their Fourier transforms

$$
H_{i}(f)=\mathbf{F T}\left[h_{i}(t)\right]
$$

The frequency responses $H_{i}(f)$ represent the frequency dependency of the higher-order components. $H_{i}(f)$ may be regarded as the system frequency response, when considering only the effect of the input frequency $f$ on the $i$ th harmonic frequency of the output. The theoretical background of the Synchronized Swept-Sine method is detailed in [10].

\section{Model Identification}

In this section, the frequency responses $H_{i}(f)$ described in the previous section are used for a nonlinear model based on a multiple-input single-output (MISO) model [3]. The structure of this model is shown in Figure 2. It is made up of $N$ parallel branches, each branch consisting of a linear filter $A_{n}(f)$. The input signals $g_{n}[x(t)]$ are known as linear and/or nonlinear functions of $x(t)$ chosen by the user.

The output signal $y(t)$ of the nonlinear system can then be expressed as

$$
y(t)=\sum_{n=1}^{N} \int_{-\infty}^{\infty} g_{n}[x(\tau)] a_{n}(t-\tau) d \tau,
$$




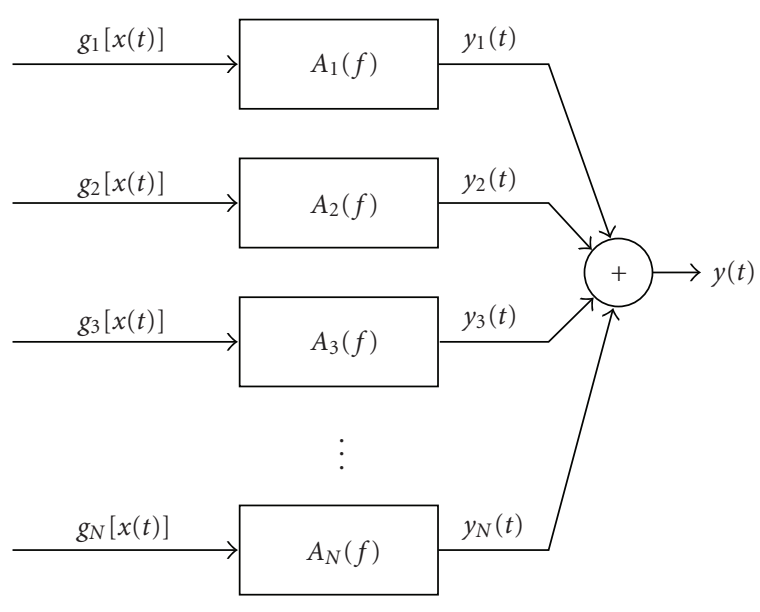

FIGURE 2: MISO model for nonlinear system identification with the input signals $g_{n}[x(t)]$ and the linear filters $A_{n}(f), n \in[1, N]$.

where $N$ is the number of the input signals of the MISObased nonlinear model, and where $a_{n}(t)$ is the impulse response related to the $n$th branch of the MISO based nonlinear model

$$
a_{n}(t)=\mathbf{F T}^{-1}\left[A_{n}(f)\right] .
$$

The linear filters $A_{n}(f)$ (or equivalently the impulse responses $a_{n}(t)$ ) have then to be identified, using the previously estimated $H_{i}(f)$. This identification consists in solving a linear system of $N$ equations using the least-squares method. First, the coefficients $c_{n, k}$ of Discrete Fourier Series of the functions $g_{n}[x(t)]$ are calculated as

$$
c_{n, k}=\frac{2}{M} \sum_{m=0}^{M-1} g_{n}\left[\sin \left(\frac{2 \pi}{M} m\right)\right] \exp \left(-j \frac{2 \pi}{M} k m\right),
$$

for an input signal being a discrete-time harmonic signal of length $M$. Next, the following set of linear equations with unknown $A_{n}(f)$ is solved

$$
H_{i}(f)=\sum_{n=1}^{N} A_{n}(f) c_{n, i}+\operatorname{Res}(f),
$$

for $i \in[1, I]$ ( $I$ being the number of harmonics taken into account), $n \in[1, N]$, and $\operatorname{Res}(f)$ being the residue. As $I \geq N$, there can be more equations than unknowns. To solve the set of equations (8) for $I>N$, the least-squares algorithm [13] is applied, minimizing the residue $\operatorname{Res}(f)$.

If the functions $g_{n}[x(t)]$ are improperly chosen and/or if at least one of the input signals is missing, the value of the residue increases drastically, which makes $\operatorname{Res}(f)$ an $a$ posteriori criterion for the choice of the input signals $g_{n}[x(t)]$.

If one of the nonlinear functions $g_{n}[x(t)]$ produces high harmonic distortion components, nonlinear aliasing [14] can appear. This can be avoided by choosing the nonlinear functions $g_{n}[x(t)]$ according to any mathematical series. The most used series is the one based on the power series, such as

$$
g_{n}[x(t)]=x^{n}(t) .
$$

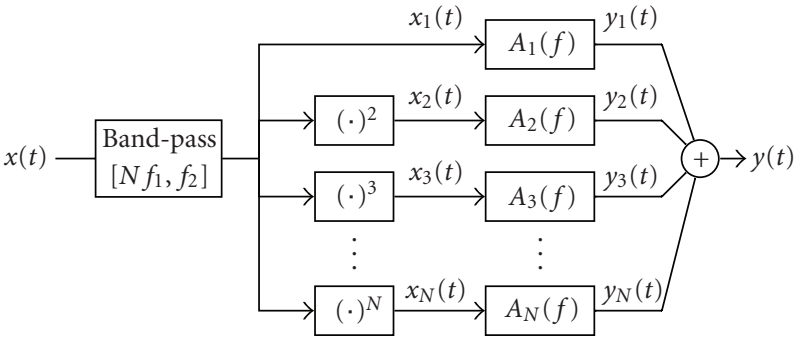

Figure 3: Generalized Polynomial Hammerstein (GPH) model (power series nonlinear model) for nonlinear system identification.

A model with inputs chosen as power series is equivalent to the Generalized Polynomial Hammerstein (GPH) model [15] with $N$ branches. In such a case, the nonlinear aliasing can be controlled by the frequency range. The highest frequency must not exceed $f_{s} /(2 N)$, where $f_{s}$ is the sampling frequency. The lowest frequency limit is as well given by the highest power function $N$. The filters $A_{n}(f)$ are indeed valid only in the frequency band $\left[N f_{1}, f_{2}\right]$. For that reason, the model should be preceded by a bandpass filter as shown in Figure 3. The amplitude limitation is as well given by the excitation signal $x_{s}(t)$ used for the analysis. As the nonlinear system is tested using an excitation signal $x_{s}(t)$, the level of which does not exceed the amplitude $A_{s}$, the nonlinear system is valid only for an input signal not exceeding $A_{s}$.

\section{Experimental Measurements: Analysis and Synthesis}

In a previous work, the Synchronized Swept-Sine method has been used to model the limiter part of a dynamic processor [10]. Results have shown the ability of the method to estimate very hard distortions with a good accuracy within the whole frequency range. In this section, the same method is tested on two real-world analog audio effects devices exhibiting weak distortions. Both devices under test are overdrive effect pedals. The first one is an Ibanez Tube Screamer ST-9 [16], the second one is a home-made overdrive pedal, the electric circuit diagram of which being depicted in Figure 4. These pedals exhibit different nonlinear performances, as investigated below.

The experimental measurement consists of two steps: (a) identification of the nonlinear system under test through the GPH model as described in the previous section and (b) comparison of the output signals of both the nonlinear system under test and the GPH model when excited with the same signal.

For the first step, the measurement setup is as follows: the sampling frequency used for the experiment is $f_{s}=192 \mathrm{kHz}$ and the excitation signal $x_{s}(t)$ is sweeping from $f_{1}=5 \mathrm{~Hz}$ to $f_{2}=10 \mathrm{kHz}$ with a maximum amplitude $A_{s}=1 \mathrm{~V}$. The filters $A_{n}(f)$ of the GPH model are then estimated.

The second step is the validation of the model for several input levels. To analyze the accuracy of the GPH model, the following test is performed. An input signal is provided to the inputs of both the real-world analog effect device and its 


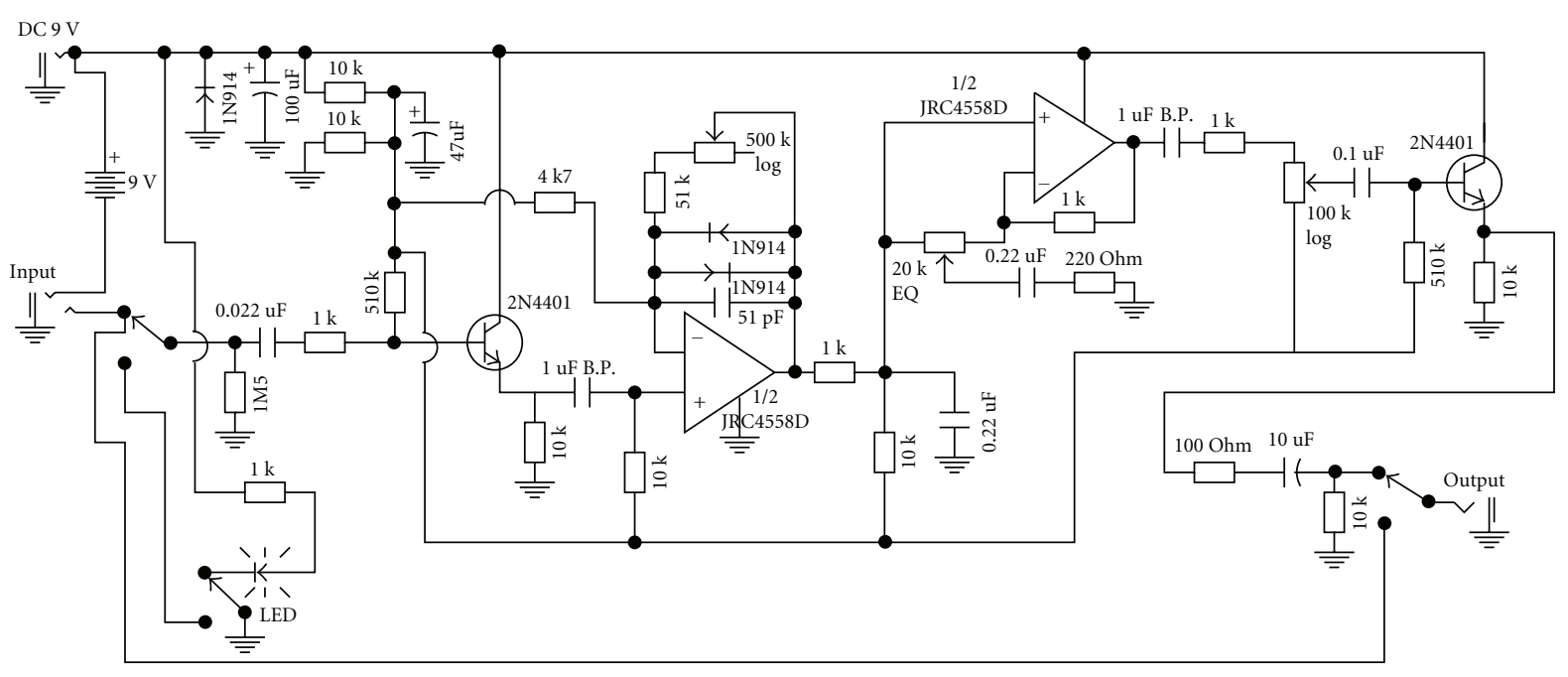

FIGURE 4: Circuit diagram of the home-made overdrive effect pedal.

corresponding GPH model, and both outputs are compared in the time and frequency domains. The input signal $x(t)$ is a sine-wave with frequency $f_{0}=500 \mathrm{~Hz}$ and amplitude $A_{0}$ that varies from $0.1 \mathrm{~V}$ to $1 \mathrm{~V}$ with step $0.1 \mathrm{~V}$. Regarding distortion measurements, we choose to test the accuracy of the method through the weighted harmonic distortion (HI2) that takes into account the higher-order components more than the classical harmonic distortion [17].

4.1. Computing Complexity versus Accuracy. The choice of the number of branches $N$ of the GPH model is a key parameter which may influence the accuracy of the identification. The higher the value of $N$ the higher the accuracy but the higher the computing complexity. To choose an optimal value of $N$, the Ibanez Tube Screamer is firstly tested for different values of $N$. Then, the HI-2 is calculated for both output signals, the output of the real-world system and the GPH model-based output, when excited with a sine wave with frequency $f_{0}=500 \mathrm{~Hz}$ and amplitude $A_{0}=1 \mathrm{~V}$.

Both the HI-2 difference, noted $\Delta \mathrm{HI}-2$ and given in $\mathrm{dB}$, and the relative computing complexity $\mathrm{CC}$ are presented in Table 1. The CC is defined as the computational time needed to generate the output of the GPH model with $N$ branches, normalized by the computational time needed to generate the output of a GPH model for $N=1$ (linear system case). (The simulation is made in Matlab for $f_{s}=192 \mathrm{kHz}$ and for a signal with a number of samples equal to $f_{s}$.)

As shown in Table 1 (for the nonlinear system under test), the choice $N=7$ is a good candidate for an optimal value between the accuracy and the computational time. Increasing $N$ does indeed not increase the accuracy of the model, but increases the computational time. As the model is made up of parallel branches (each branch including the same computing complexity), the computational time is directly proportional to $N$.

4.2. Ibanez Tube Screamer Overdrive Pedal. The first nonlinear system under test is an overdrive effect pedal Ibanez Tube
Screamer [16] (pedal 1). The pedal has been configured as follows: Drive $=4 / 10$, Level $=6 / 10$, Mid Boost $=0 / 10$, and Tone $=3 / 10$. Driving input level is attenuated by $25 \mathrm{~dB}$ before exciting the nonlinear system under test.

The outputs corresponding to an input sine wave with $f_{0}=500 \mathrm{~Hz}$ and $A_{0}=1 \mathrm{~V}$ are shown in Figure 5, in both time and frequency domains. The HI-2 is $-21.14 \mathrm{~dB}$ for the real-world output and $-21.17 \mathrm{~dB}$ for the model output $(\mathrm{N}=$ 7 ), that illustrates a very good accuracy of the identification method.

The HI-2 for both model and real-world system are compared in Figure 6, when measured with increasing input signal level $A_{0}$ (from $0.1 \mathrm{~V}$ to $1 \mathrm{~V}$ ). The HI-2 fits only for the maximum input level $A_{0}=1 \mathrm{~V}$, corresponding to the level $A_{s}$ of the signal used for the estimation of the $A_{n}(f)$. For other levels $A_{0}<1 \mathrm{~V}$, discrepancies between the HI-2 spread from 4 to $8 \mathrm{~dB}$. For example, the responses of the GPH model and the real-world device to a sine wave, the amplitude of which is $A_{0}=0.5 \mathrm{~V}$, is given in Figure 7, in both time and frequency domains. The regenerated output signal, based on the GPH model estimated for $A_{s}=1 \mathrm{~V}$, does not fit with the real-world output signal. The HI-2 is $-28.0 \mathrm{~dB}$ for the real-world output and $-34.6 \mathrm{~dB}$ for the model output.

As a consequence, the nonlinear system under test (Ibanez Tube Screamer) can be seen as a nonlinear system whose input/output law is driven by the input level $A_{0}$. Nevertheless, when the amplitude $A_{0}$ of the input signal is the same as the amplitude $A_{s}$ used for the identification of the nonlinear system, the GPH model-based output fits well with the real-world output. This is illustrated in Figure 8 for the case $A_{s}=A_{0}=0.5 \mathrm{~V}$. The HI-2 is then $-28.0 \mathrm{~dB}$ for the real-world output and $-27.9 \mathrm{~dB}$ for the GPH model-based output.

4.3. Home-Made Overdrive Pedal. The second nonlinear system under test is a home-made overdrive pedal, noted pedal 2, exhibiting lower dependency on input level. The circuit diagram of the pedal 2 is presented in Figure 4. 
TABLE 1: Effect of the number of branches $N$ of the GPH model on the weighted harmonic distortion difference $\Delta \mathrm{HI}-2$ and on the relative computing complexity CC.

\begin{tabular}{lccccccccccc}
\hline$N$ & 1 & 2 & 3 & 4 & 5 & 6 & 7 & 8 & 9 & 10 & 11 \\
\hline$\Delta \mathrm{HI}-2[\mathrm{~dB}]$ & $-\infty$ & 39.8 & 1.9 & 1.9 & 0.31 & 0.31 & 0.03 & 0.03 & 0.03 & 0.03 & 0.03 \\
$\mathrm{CC}[-]$ & 1.0 & 2.0 & 3.1 & 4.2 & 5.4 & 6.5 & 7.5 & 8.6 & 9.7 & 10.7 & 11.9 \\
\hline
\end{tabular}

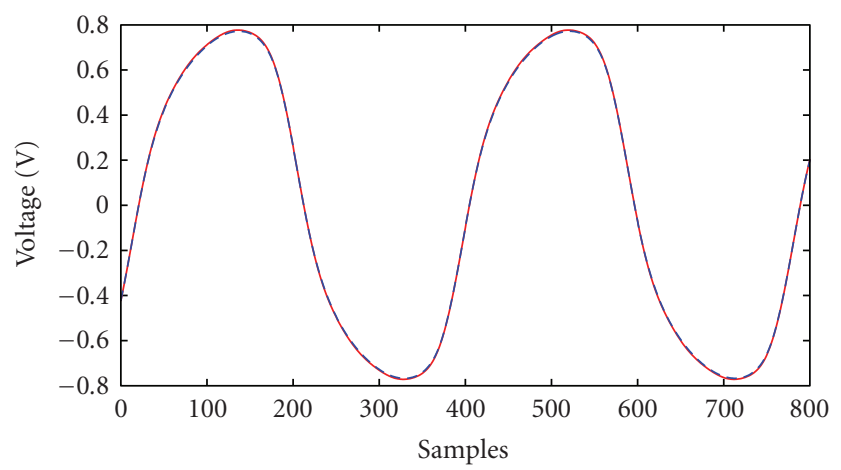

(a)

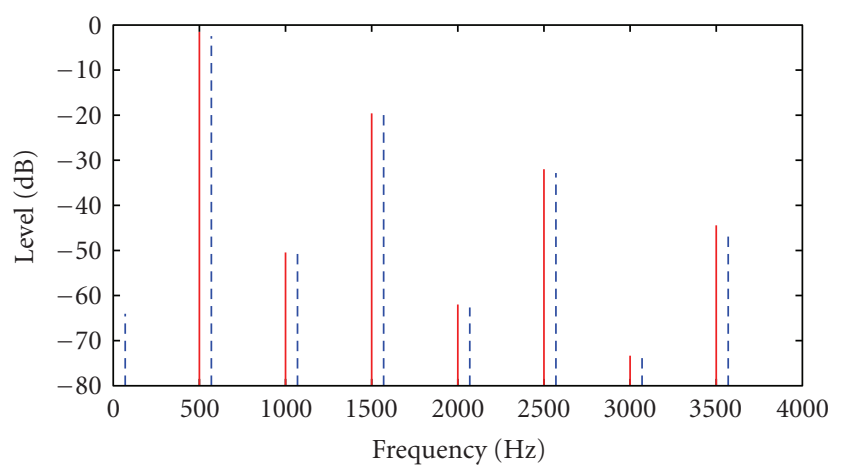

(b)

FIGURE 5: Comparison between the real-world output of pedal 1 (blue-dashed) and the GPH model-based output (red-solid) in time (a) and frequency (b) domains, for a sine wave excitation with $f_{0}=500 \mathrm{~Hz}$ and $A_{0}=1 \mathrm{~V}$. The GPH model is estimated using a swept sine signal with amplitude $A_{s}=1 \mathrm{~V}$. For the sake of clarity, the output of the real-world device in the frequency domain is shifted to the right.

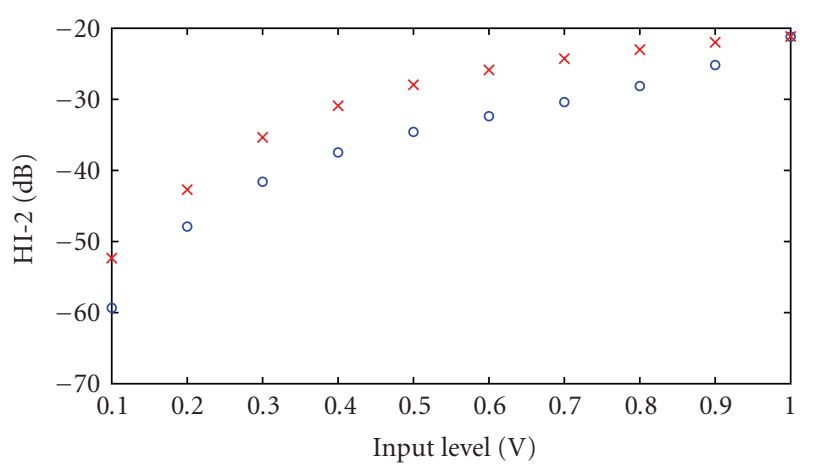

- Real-world

$\times$ Synthesized

FIGURE 6: HI-2 of synthesized and real-world signals as a function of the input level (pedal 1).

The same configuration and analysis as those described in Section 4.2 have been setup.

The outputs corresponding to an input sine wave of $f_{0}=$ $500 \mathrm{~Hz}$ and $A_{0}=1 \mathrm{~V}$ are shown in Figure 9. The HI-2 is $-29.56 \mathrm{~dB}$ for the real-world output and $-29.45 \mathrm{~dB}$ for the GPH model output. As for the case of pedal 1, it illustrates a very good accuracy of the identification method. The outputs corresponding to an input sine wave of $f_{0}=500 \mathrm{~Hz}$ and $A_{0}=0.5 \mathrm{~V}$ (Figure 10) show also a good agreement even if the amplitude $A_{0}$ of the input signal differs of the amplitude $A_{s}=1 \mathrm{~V}$ used for the identification of the nonlinear system. The HI- 2 is $-45.0 \mathrm{~dB}$ for the real-world output and $-43.2 \mathrm{~dB}$ for the model output.
As illustrated in Figure 11, the difference $\Delta \mathrm{HI}-2$ between both HI-2 is less than $2.5 \mathrm{~dB}$ for all the input levels $A_{0}$. Thus, such a nonlinear system represents a system whose input/output law is not driven by the input level $A_{0}$. For such a nonlinear system, the presented identification method with GPH model can be used for both analysis and synthesis.

\section{Classification of Input Level (In)Dependent Nonlinear Systems}

In the previous section, two real-world nonlinear systems, exhibiting different nonlinear behaviors have been identified thanks to the Synchronized Swept Sine method. The input/output law of the first system under study (pedal 1) is driven by the input level $A_{0}$, while the input/output law of the second one (pedal 2) is independent of this input level. In the following, we call "input level dependent" the first kind of nonlinear system and "input level independent" the second one.

A key point of the method of identification presented in this paper is its capacity to distinguish both kinds of nonlinear systems through its ability to synthesize the output signals from any given input signal. Then, the classification of nonlinear systems in these two categories (input level dependent and input level independent) is performed here thanks to the Synchronized Swept-Sine method. A criterion based on the analysis of impulse responses $a_{n}(t)$ of GPH model is used to perform this classification.

More specifically, we show that analyzing only the first branch (linear part) of the model is sufficient to classify both kinds of nonlinear systems. The linear impulse response $a_{1}(t)$ is firstly estimated for $N=7$ and for several input levels $A_{s} \in$ $[0.1,1] \mathrm{V}$, noted $a_{1, l}(t) ; l$ denoting the input of the index 


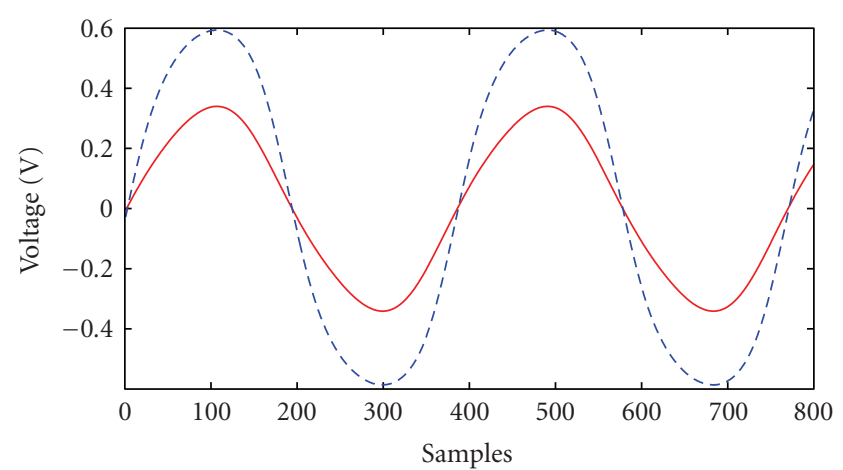

(a)

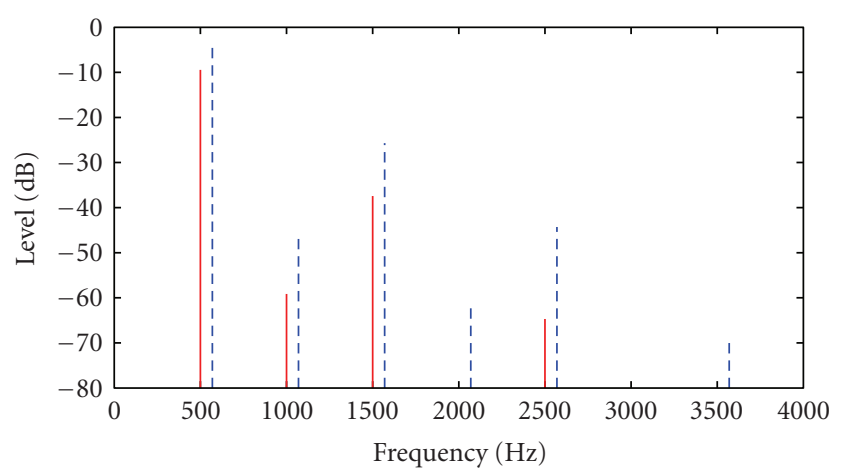

(b)

FIGURE 7: Comparison between the real-world output of pedal 1 (blue-dashed) and the GPH model-based output (red-solid) in time (a) and frequency (b) domains, for a sine wave excitation with $f_{0}=500 \mathrm{~Hz}$ and $A_{0}=0.5 \mathrm{~V}$. The GPH model is estimated using a swept sine signal with amplitude $A_{s}=1 \mathrm{~V}$. For the sake of clarity, the output of the real-world device in the frequency domain is shifted to the right.

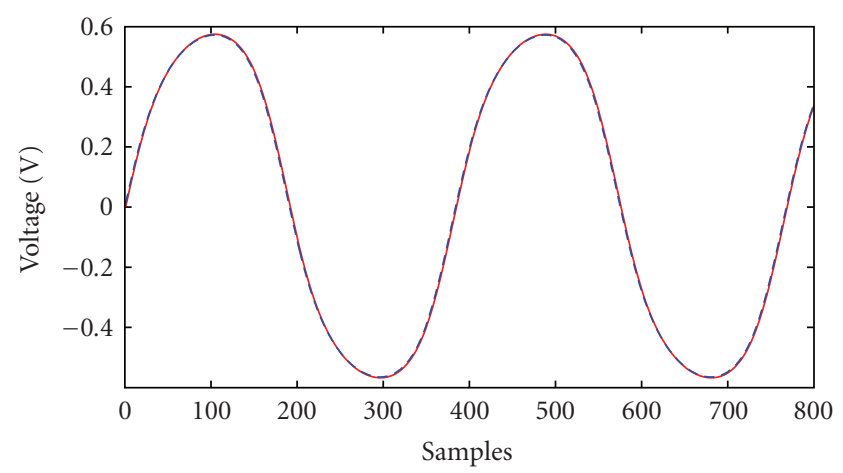

(a)

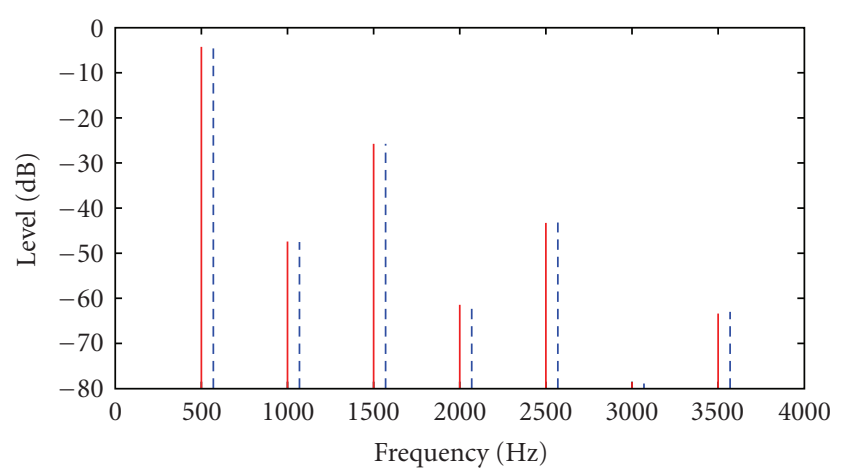

(b)

FIGURE 8: Comparison between the real-world output of pedal 1 (blue-dashed) and the GPH model-based output (red-solid) in time (a) and frequency (b) domains, for a sine wave excitation with $f_{0}=500 \mathrm{~Hz}$ and $A_{0}=0.5 \mathrm{~V}$. The GPH model is estimated using a swept sine signal with amplitude $A_{s}=0.5 \mathrm{~V}$. For the sake of clarity, the output of the real-world device in the frequency domain is shifted to the right.

level. Then, if the nonlinear system under test is an "input level dependent" one, the impulse responses are expected to be different from each other. On the contrary, if the nonlinear system under test is an "input level independent" one, the impulse responses $a_{1, l}(t)$ are expected to be very close each other.

In Figures 12 and 13, the impulse responses $a_{1, l}(t)$ of the first branch of the nonlinear model are depicted for different input levels, for the case of pedals 1 and 2 respectively. Using these results, we propose to define the following relative squared error (RSE) based criterion for classifying the nonlinear systems under test,

$$
\operatorname{RSE}_{l}=\frac{\int\left\{a_{1, l}(t)-\left\langle a_{1}(t)\right\rangle\right\}^{2} d t}{\int\left\langle a_{1}(t)\right\rangle^{2} d t},
$$

where $\left\langle a_{1}(t)\right\rangle$ is the average impulse response,

$$
\left\langle a_{1}(t)\right\rangle=\frac{\sum_{l=1}^{10} a_{1, l}(t)}{10} .
$$

The RSE measures the mean-squared distance between the average impulse response $\left\langle a_{1}(t)\right\rangle$ and the impulse responses $a_{1, l}(t)$.
For the case of pedal 1, we have $\max \left(\mathrm{RSE}_{l}\right)=10 \%$, whilst for the case of pedal $2, \max \left(\mathrm{RSE}_{l}\right)=1.3 \%$. This order of magnitude between both values clearly allows to classify the input level dependent and input level independent nonlinear systems under test.

\section{Conclusions}

In this paper, a recently proposed method [10] is tested for classifying, analyzing, and synthesizing two nonlinear systems (overdrive effect pedals) exhibiting different nonlinear behaviors. The method for identification of nonlinear systems is based on synchronized swept-sine signal and allows the identification of nonlinear system under test in a one-path measurement.

The classification is indispensable for distinguishing nonlinear systems whose input/output law is driven by input level, and nonlinear systems whose input/output law is independent of the input level.

Two nonlinear systems have been tested: the first one corresponding to a nonlinear system whose input/output law is driven by the input level and the second being 


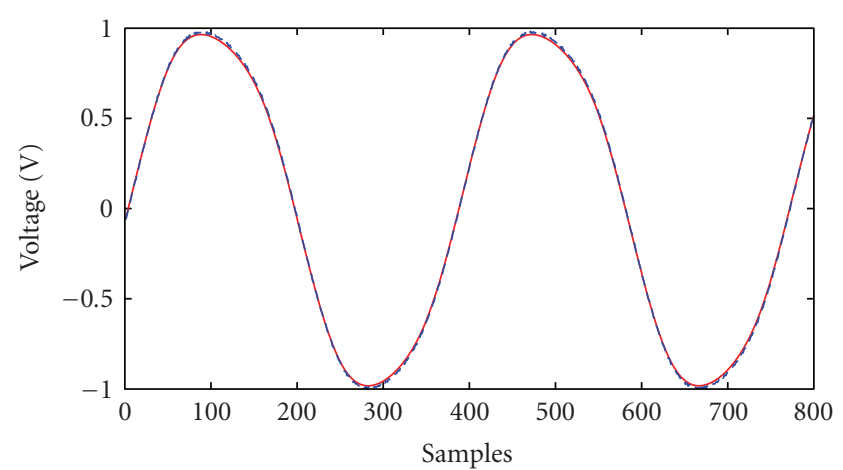

(a)

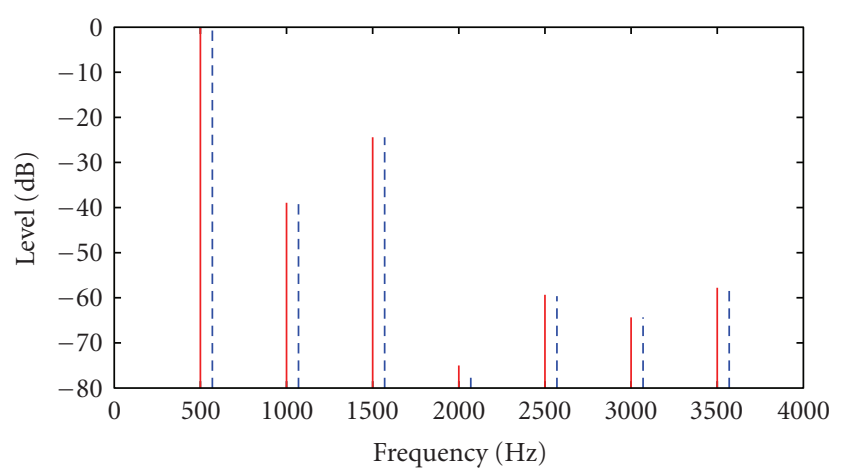

(b)

FIGURE 9: Comparison between the real-world output of pedal 2 (blue-dashed) and the GPH model-based output (red-solid) in time (a) and frequency (b) domains, for a sine wave excitation with $f_{0}=500 \mathrm{~Hz}$ and $A_{0}=1 \mathrm{~V}$. The GPH model is estimated using a swept sine signal with amplitude $A_{s}=1 \mathrm{~V}$. For the sake of clarity, the output of the real-world device in the frequency domain is shifted to the right.

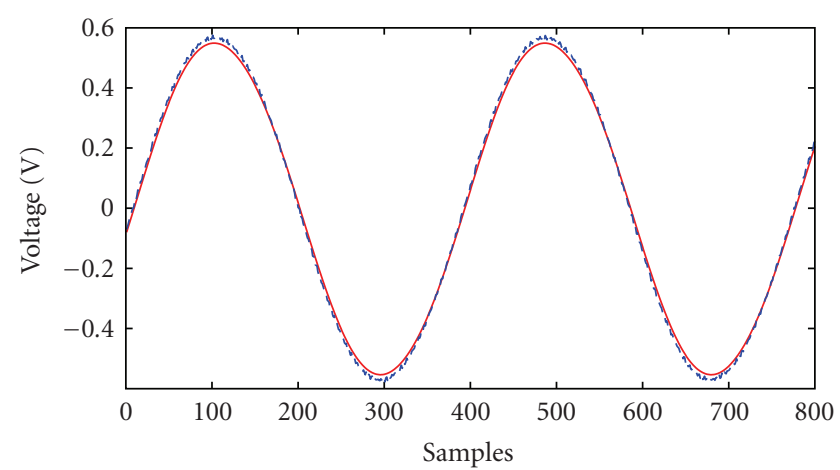

(a)

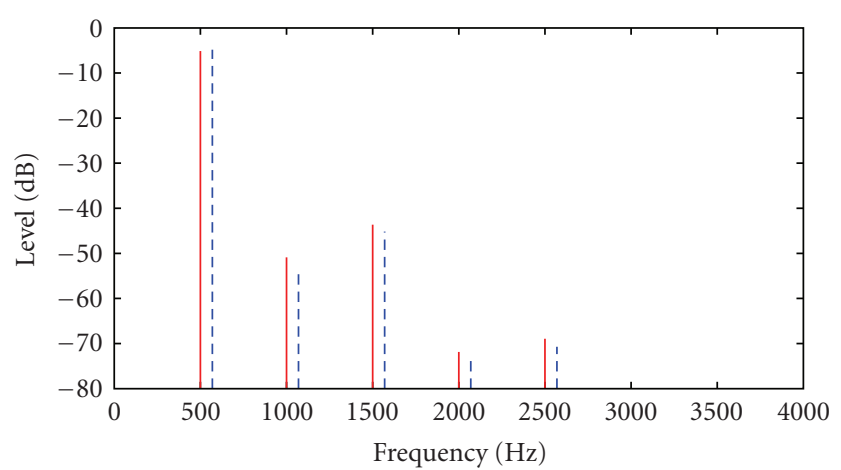

(b)

FIGURE 10: Comparison between the real-world output of pedal 2 (blue-dashed) and the GPH model-based output (red-solid) in time (a) and frequency (b) domains, for a sine wave excitation with $f_{0}=500 \mathrm{~Hz}$ and $A_{0}=0.5 \mathrm{~V}$. The GPH model is estimated using a swept sine signal with amplitude $A_{s}=1 \mathrm{~V}$. For the sake of clarity, the output of the real-world device in the frequency domain is shifted to the right.

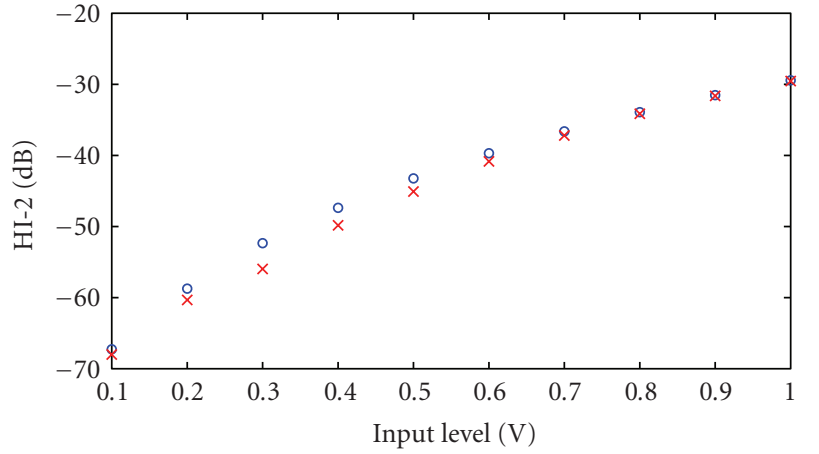

- Real-world

$\times$ Synthesized

FIGURE 11: HI-2 of synthesized and real-world signals as a function of the input level (pedal 2).

a nonlinear system whose input/output law is independent of the input level. For the latter (pedal 2), the results show that the method is useful for both analysis and synthesis. The comparison between the synthesized and

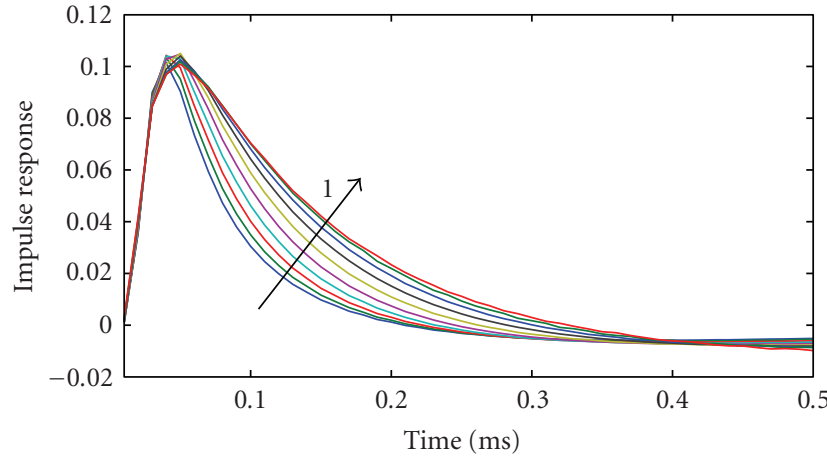

FIGURE 12: Impulse responses $a_{1, l}(t)$ of the first branch of the nonlinear model $(N=7)$ depicted for different input levels (pedal $1)$.

real-world signal shows very good agreement in both time and frequency domains. The same agreement is shown by comparing the weighted harmonic distortion HI-2 [17]. 


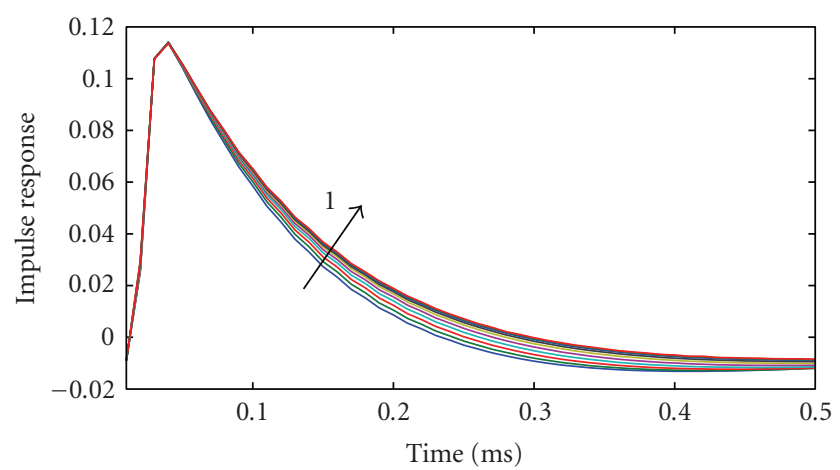

FIGURE 13: Impulse responses $a_{1, l}(t)$ of the first branch of the nonlinear model $(N=7)$ depicted for different input levels (pedal 2).

In the case of input level dependent nonlinear system (pedal 1), it is shown that when the identification is carried out from a signal with input level $A_{s}$, the model is very accurate only when the amplitude of the input signal to be synthesized is $A_{0}=A_{s}$. Thus, for a whole analysis of such a system, the frequency responses $H_{i}(f)$ have then to be estimated for different input levels $A_{s}$, leading to $2 \mathrm{D}$ frequency response functions (FRF) $H_{i}\left(f, A_{s}\right)$.

Works are now in progress to implement the FRF $H_{i}\left(f, A_{s}\right)$ into the nonlinear model in order to synthesize such systems for any input signals.

\section{Acknowledgments}

This work was supported by the French region Pays de la Loire. The authors would like to thank J. B. Doc and J. C. Le Roux for their help in selecting two overdrive pedals, and to the anonymous reviewers for their helpful comments.

\section{References}

[1] M. Schetzen, The Volterra and Wiener Theories of Nonlinear Systems, John Wiley \& Sons, New York, NY, USA, 1980.

[2] O. Nelles, Nonlinear System Identification: From Classical Approaches to Neural Networks and Fuzzy Models, Springer, Berlin, Germany, 2001.

[3] H. Kusaka, M. Kominami, K. Matsumoto, and Y. Tashiro, "Performance improvement of CDMA/QPSK systems with nonlinear channel using decision feedback equalizers," in Proceedings of the 5th IEEE International Symposium on Spread Spectrum Techniques \& Applications, pp. 812-817, September 1998.

[4] F. Thouverez and L. Jezequel, "Identification of NARMAX models on a modal base," Journal of Sound and Vibration, vol. 189, no. 2, pp. 193-213, 1996.

[5] Y.-W. Chen, S. Narieda, and K. Yamashita, "Blind nonlinear system identification based on a constrained hybrid genetic algorithm," IEEE Transactions on Instrumentation and Measurement, vol. 52, no. 3, pp. 898-902, 2003.

[6] H. W. Sorenson, Kalman Filtering: Theory and Application, IEEE Press, Montvale, NJ, USA, 1985.

[7] O. Cappe, S. J. Godsill, and E. Moulines, "An overview of existing methods and recent advances in sequential Monte
Carlo," Proceedings of the IEEE, vol. 95, no. 5, pp. 899-924, 2007.

[8] A. Farina, A. Bellini, and E. Armelloni, "Non-linear convolution: a new approach for the auralization of distorting systems," in Proceedings of the 110th Audio Engineering Society Convention, Amsterdam, The Netherlands, May 2001.

[9] A. Farina, "Simultaneous measurement of impulse response and distortion with a swept-sine technique," in Proceedings of the 108th Audio Engineering Society Convention, Paris, France, February 2000.

[10] A. Novák, L. Simon, F. Kadlec, and P. Lotton, "Nonlinear system identification using exponential swept-sine signal," IEEE Transactions on Instrumentation and Measurement, vol. 59, no. 8, pp. 2220-2229, 2010.

[11] U. Zölzer, DAFX: Digital Audio Effects, John Wiley \& Sons, New York, NY, USA, 2002.

[12] G. Bertotti and I. D. Mayergoyz, The Science of Hysteresis, Academic Press, Boston, Mass, USA, 2006.

[13] G. Strang, Introduction to Applied Mathematics, WellesleyCambridge Press, Cambridge, Mass, USA, 1986.

[14] Y. Zhu, "Generalized sampling theorem," IEEE Transactions on Circuits and Systems II, vol. 39, no. 8, pp. 587-588, 1992.

[15] A. Janczak, Identification of Nonlinear Systems Using Neural Networks and Polynomial Models: A Block-Oriented Approach, Springer, Berlin, Germany, 2005.

[16] Ibanez ST 9, "Super Tube Screamer," 2007, http://www .ibanez.com/Electronics/model-TS9DX.

[17] W. Klippel, "Measurement of Weighted Harmonic Distortion HI-2," in Application Note, Klippel GmbH, 2002. 